FOLIA

Amazónica

Revista del Instituto de Investigaciones

de la Amazonía Peruana

\title{
USOS DE FAUNA SILVESTRE EN EL TRAPECIO AMAZÓNICO COLOMBIANO Y APORTES HACIA SU GESTIÓN INTEGRAL EN EL DEPARTAMENTO DE AMAZONAS
}

\author{
Astrid Fernanda HERNÁNDEZ-CORONADO ${ }^{1}$ \\ 1 Corporación para el desarrollo sostenible del sur de la Amazonia-Corpoamazonia \\ dendrobaf@hotmail.com
}

\section{RESUMEN}

Se identificaron diferentes usos de fauna silvestre en nueve localidades rurales (comunidades indígenas y colonos) ubicadas en el Trapecio Amazónico Colombiano, a partir de la aplicación de 239 entrevistas semi-estructuradas. Se presentan las especies más utilizadas y con mayor presión de caza a través de sus experiencias alimenticias, mágico-religiosas y costumbres. En total se identificaron 73 especies de fauna silvestre (35 de mamíferos, 23 de aves, 11 de reptiles, 2 de coleópteros y 1 de gasterópodo). El principal uso de la fauna es la alimentación, seguido por el uso medicinal y como animales de compañía. Las partes de animales son utilizadas para actividades mágico-religiosas, artesanías, instrumentos musicales, herramientas y atuendos. Como producto final se desarrolla una propuesta de gestión de fauna silvestre para el trapecio amazónico.

PALABRAS CLAVE: Usos, especies, Trapecio Amazónico, fauna. 


\title{
USES OF WILDLIFE IN THE COLOMBIAN AMAZON TRAPEZE AND CONTRIBUTIONS TOWARDS ITS INTEGRAL MANAGEMENT IN THE AMAZON DEPARTMENT
}

\begin{abstract}
The different wildlife uses were identified in nine rural localities (indigenous communities and settlers) located in the Colombian Amazonian Trapeze, from the application of 239 semi-structured interviews. The species most used and most hunting pressure through their food experiences, magic-religious, and customs are presented. In total, we identified 73 species of wildlife (35 of mammals, 23 of birds, 11 of reptilian, 2 of coleoptera and 1 of gastropod). The main use of wildlife is feeding, followed by medicinal use and as pets. Parts of animals are used for magic-religious activities, crafts, musical instruments, tools and costumes. It as final product develops a proposal of management of wildlife for the Amazonian trapezium.
\end{abstract}

KEYWORDS: Uses, species, Amazonian trapezium, wildlife. 


\section{INTRODUCCIÓN}

Colombia es el segundo país megadiverso del mundo por el número de especies registradas. De acuerdo con el SiB Colombia (2016), se reportan 1921 especies de aves, de las cuales, alrededor de 79 son endémicas; 492 especies de mamíferos; 803 especies de anfibios; 537 especies de reptiles y 15.814 especies de insectos. La fauna silvestre amazónica es patrimonio natural de un gran valor e importancia mundial debido a la diversidad de especies, a las múltiples funciones ecológicas que cumple, y a su aporte como un importante servicio ecosistémico para la subsistencia y la continuidad ecosistémica y cultural de las comunidades humanas (Baptiste et al. 2002, Cruz-Antia \& Gómez, 2010,), como ocurre en las áreas rurales del departamento de Amazonas, que es mayoritariamente de población indígena.

El uso de la fauna silvestre en la región amazónica se remonta al origen del habitante amazónico hace aproximadamente 10.000 años, donde utilizaron los animales como alimento y dieron valor a productos como las plumas, huevos, pieles, entre otros, usados para rituales tradicionales, productos mágicos $\mathrm{o}$ curativos, ornamentos y vestimentas (Tratado de Cooperación Amazónica-TCA, 1999). En la región amazónica existen estudios que demuestran que los grupos indígenas utilizan variedad de animales silvestres a lo largo del año, entre los que se destacan mamíferos, aves y reptiles (De la Hoz 2007, Osorno et al., 2014)

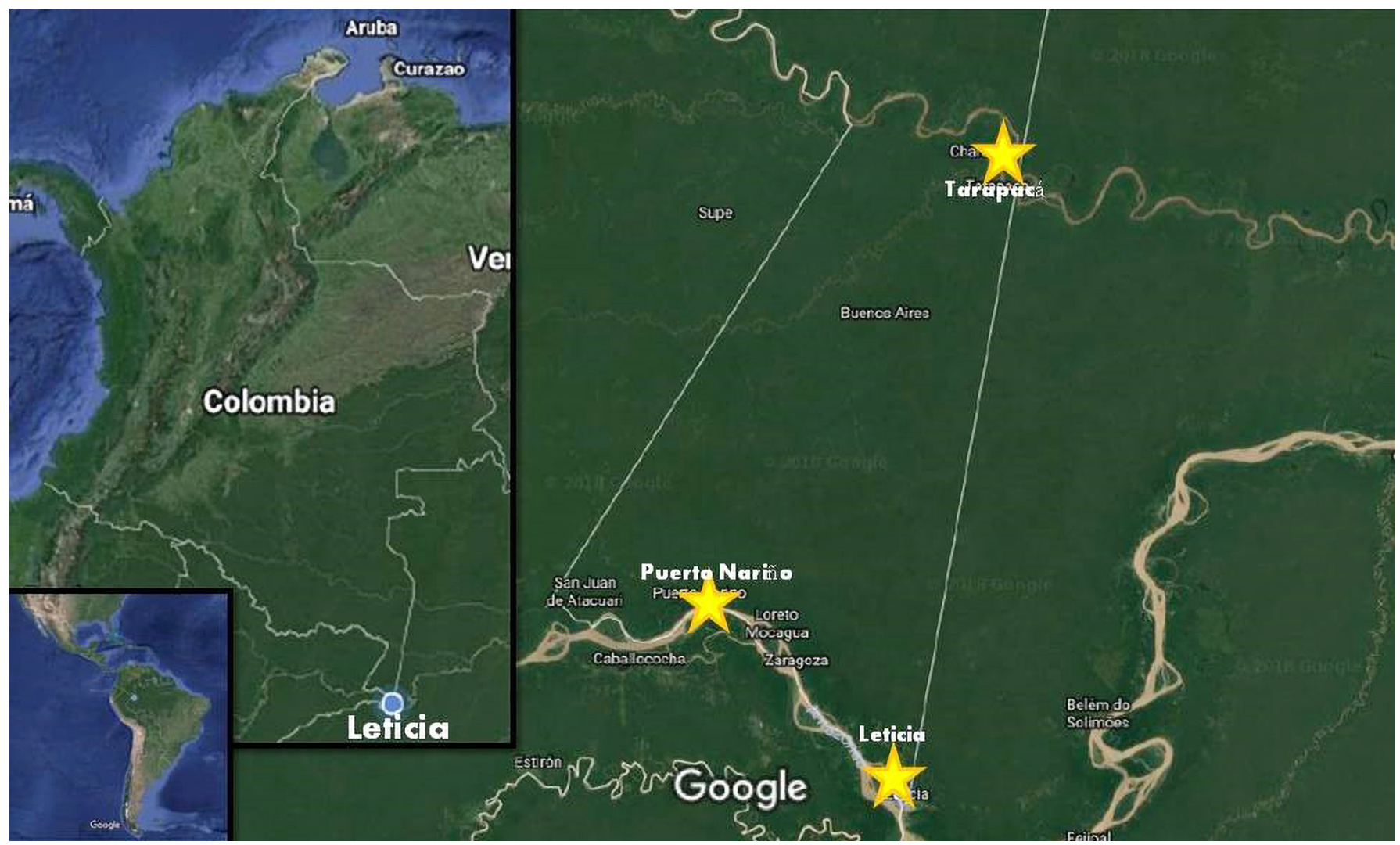

Figura 1. Localización geográfica de la zona de estudio. Los puntos amarillos demarcan el área de estudio. Imagen obtenida de Google (2018) Recuperada de https://www.google.com.co/maps/@-3.4231837,-70.2937594,203952m/ data $=! 3 \mathrm{~m} 1 ! 1 \mathrm{e} 3$ 
El rescate de los conocimientos o saberes locales se ubica como una prioridad en la agenda ambiental del nuevo milenio del informe sobre Ecosistemas y Bienestar del Hombre (Millennium Ecosystem Assessment, 2005), por esta razón, el estudio de los usos de este recurso natural a nivel comunitario constituye uno de los instrumentos de análisis para la toma de decisiones concernientes a favorecer el aprovechamiento sustentable de las comunidades indígenas y colonos que habitan la región. Con este argumento, se desarrolló esta investigación que tiene como objetivo conocer los usos de la fauna silvestre en las comunidades rurales del Trapecio Amazónico Colombiano. Información que permitirá avanzar en la gestión de la fauna silvestre en esta parte de la región amazónica y que se convierte en uno de los principales aportes de este estudio.

\section{MATERIALES Y MÉTODOS}

Lainvestigación se desarrolló en nueve localidades ubicadas en el Trapecio Amazónico Colombiano (Figura 1), que está compuesto por dos municipios (Leticia y Puerto Nariño) y Tarapacá, una área no municipalizada (Ruiz \& Valencia 2007), con asentamientos humanos urbanos, donde la mayor parte de las comunidades indígenas se encuentran en zonas rurales.

EnelMunicipiodeLeticia,seaplicaronencuestas en siete comunidades rurales (Macedonia, San Antonio de Los Lagos, San José-Kilómetro 6, Kilometro 11, San Miguel-Calderón, Nazareth), y dos en el perímetro urbano correspondientes a Puerto Nariño y Tarapacá.

Se aplicaron encuestas semi-estructuradas puerta a puerta, a una persona por núcleo familiar en las comunidades objeto de estudio, durante el mes de julio de 2015 a marzo del 2016, donde se recolectó información sobre las especies empleadas y los diferentes usos dados por sus hábitos alimenticios y costumbres ancestrales. El análisis y validación de los resultados de las encuestas aplicadas se hizo de forma práctica e interpretativa y se aplicaron los planteamientos del Análisis de Contenido. Este es un método empleado para estudiar comunicaciones orales y escritas demanerasistemática, objetiva,cualitativa y cuantitativa, que permite medir las variables bajo estudio y transformar un texto primitivo sobre el que se ha operado aquella transformación para modificarlo (controladamente) según las reglas de procedimiento, de análisis y de refutación (metodología) confiables y válidas, y que se hayan justificado metodológicamente (Piñuel 2002, Barrasa 2012).

\section{RESULTADOS}

En total se entrevistaron a 239 personas en nueve localidades diferentes del Trapecio Amazónico Colombiano, la mayoría indígenas, con registros de personas colonas nacionales y extranjeras que viven en estas comunidades.

En general, los mayores registros provienen de indígenas de la etnia Ticuna, seguida de la etnia Cocama y Yaguas. En las comunidades acentuadas sobre Leticia y Puerto Nariño predominan los Ticuna, mientras que los encuestados pertenecientes a las etnias Tanimuca, Yucuna y Miraña en el municipio de Leticia manifestaron provenir de áreas no municipalizadas (conocidas como corregimientos). De los encuestados, el 59\% correspondió al género femenino y el $41 \%$ al género masculino.

\section{USOS DE LA FAUNA SILVESTRE}

El principal uso de la fauna silvestre en el Trapecio Amazónico Colombiano es la alimentación (230 encuestados consumen o consumieron carne de animales silvestres, lo que corresponde al 96,2\% 


\section{USOS DE LA FAUNA SILVESTRE POR COMUNIDADES}

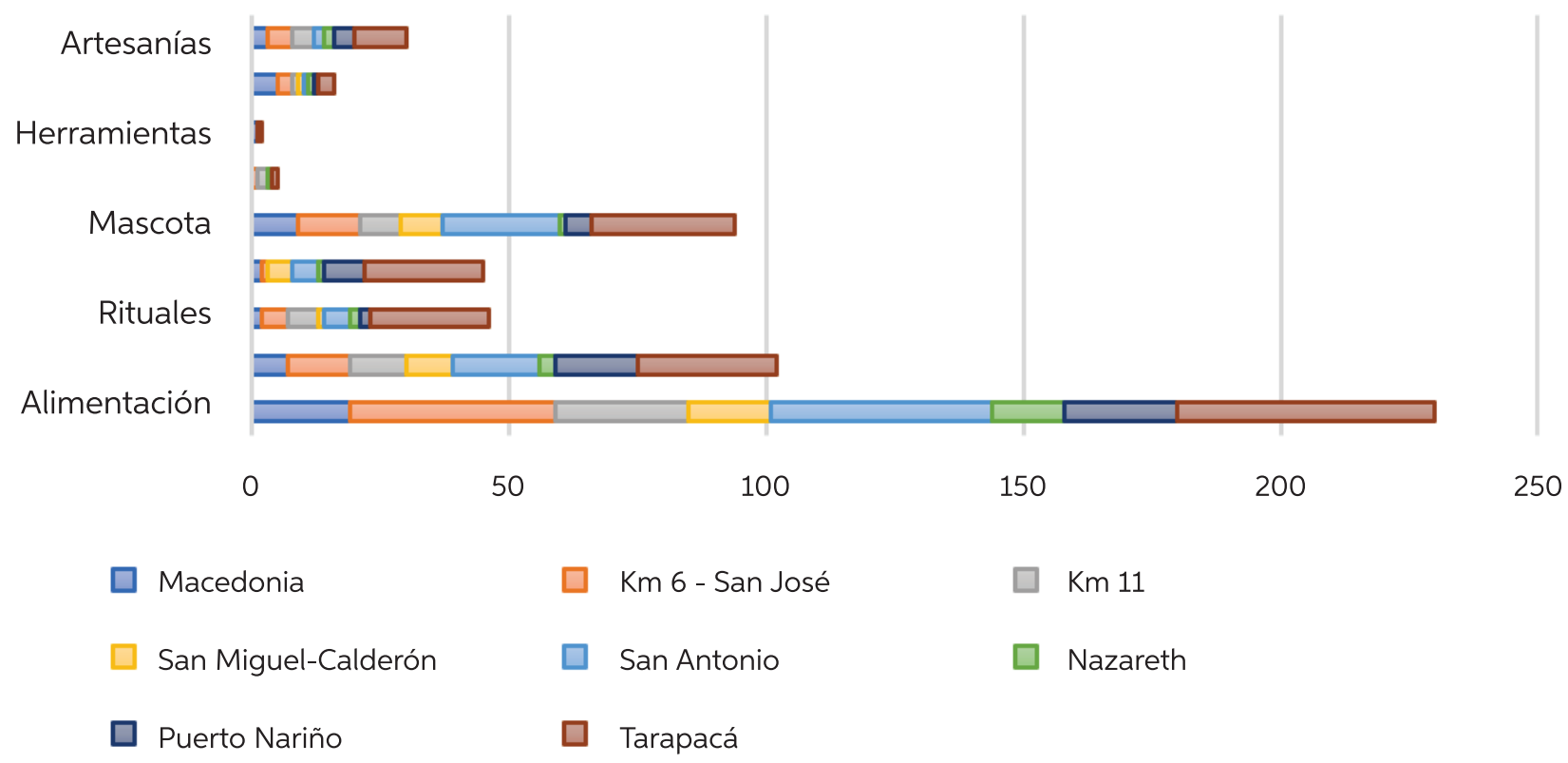

Figura 2. Usos de la fauna silvestre identificados por cada comunidad estudiada en el Trapecio Amazónico.

del total de la población) donde utilizan variedad de especies para el consumo. El uso de animales con fines medicinales todavía es practicado, aunque solamente el $42,7 \%$ de los encuestados manifestaron tener conocimiento referente al tema. Se identificaron además otros usos como animales para mascotas, donde el 39,3\% de los encuestados tiene o ha tenido un animal silvestre de compañía; por su parte, el 19,2 \% ha utilizado o conoce qué partes de animales se emplean para rituales o actividades mágico religiosos.

Para la fabricación de artesanías se utilizan partes como huesos, plumas, colmillos, garras, piel, espinas y partes de insectos; según el 12,6\% de los participantes, tan solo el 9,6\% conoce o ha utilizado partes de animales para la elaboración de instrumentos musicales, herramientas o atuendos. El 18,8\% de los encuestados manifestó haber comercializado con ejemplares vivos, partes de fauna y carne como fuente de ingreso económico.

La comunidad que mayor porcentaje de uso de fauna silvestre presentó en cada una de las categorías definidas corresponde al área no municipalizada de Tarapacá (Figura 2), donde además los diferentes usos se comunicaron abiertamente a los encuestadores, a diferencia de otros lugares, donde existió cierta restricción de los encuestados a contestar las preguntas. Igualmente, las comunidades ubicadas sobre el eje Leticia-Tarapacá, limitan el uso de animales silvestres a la alimentación, e inclusive, manifiestan la facilidad de conseguir proteína animal proveniente de animales de granja o pescado, así como la pérdida de conocimiento y celebración de rituales mágico-religiosos en sus comunidades.

\section{ESPECIES UTILIZADAS}

En total se identificaron 73 especies de fauna silvestre usadas por las comunidades encuestadas, algunas especies presentan diferentes usos y otras solamente se identificó una utilidad aparente (Tabla 1). 
Tabla 1. Relación de especies de fauna silvestre utilizadas por las comunidades estudiadas en el Trapecio Amazónico.

\begin{tabular}{|c|c|c|c|c|c|c|c|c|c|}
\hline \multicolumn{10}{|c|}{ MAMÍFEROS } \\
\hline Nombre Común & Especie & 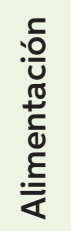 & 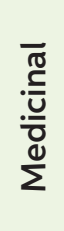 & $\frac{\tilde{d}}{\frac{\tilde{\sigma}}{\Gamma}}$ & 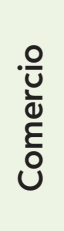 & 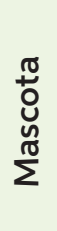 & 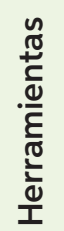 & 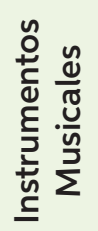 & 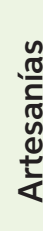 \\
\hline Churuco & Lagothrix lagothricha & $x$ & $x$ & & $x$ & $x$ & & $x$ & \\
\hline Mico volador-Huapo negro & Pithecia monachus & $x$ & $x$ & & & $x$ & & & \\
\hline Mico cariblanco & Cebus albifrons & $x$ & & & & $x$ & & & \\
\hline Mono negro & Sapajus apella & $x$ & & & & $x$ & & & \\
\hline Zogui-zogui & Callicebus sp. & $x$ & & & & & & & \\
\hline Fraile & Saimiri sciureus & $\mathrm{x}$ & & & & $x$ & & & \\
\hline Cotudo & Alouatta seniculus & $x$ & $x$ & & & $x$ & & $x$ & \\
\hline Mico nocturno & Aotus sp. & $x$ & & & & $x$ & & & $\mathrm{x}$ \\
\hline Leoncito & Cebuella pygmaea & & & & & $x$ & & & \\
\hline Bebeleche-boquileche & Saguinus sp. & & & & & $x$ & & & \\
\hline Boruga & Cuniculus paca & $x$ & $x$ & $x$ & $x$ & $x$ & & $x$ & \\
\hline Tin tin (roedor) & Myoprocta pratti & $x$ & & & & $x$ & & & \\
\hline Guara & Dasyprocta fuliginosa & $x$ & & $x$ & $x$ & $x$ & & $x$ & \\
\hline Cuzumbo & Nassua nasua & $x$ & $x$ & & & & & & \\
\hline Ratón de monte & Caviomorpha & $x$ & & & & & & & \\
\hline $\begin{array}{l}\text { Cerrillo- saíno- pecarí de } \\
\text { collar }\end{array}$ & Pecari tajacu & $x$ & & $x$ & $x$ & $x$ & $x$ & $x$ & $x$ \\
\hline Danta & Tapirus terrestris & $x$ & $\mathrm{x}$ & $x$ & $\mathrm{x}$ & $x$ & & $x$ & \\
\hline $\begin{array}{l}\text { Puerco de monte- } \\
\text { Huangana-barbiblanco }\end{array}$ & Tayassu pecari & $x$ & & $x$ & $x$ & $x$ & & $x$ & $\mathrm{x}$ \\
\hline Venado cenizo-chonto & Mazama nemorivaga & $x$ & $x$ & $x$ & $x$ & & & $x$ & \\
\hline Venado rojo & Mazama americana & $x$ & $x$ & $x$ & $x$ & $x$ & & $x$ & \\
\hline Chigüiro- ronsoco & $\begin{array}{l}\text { Hydrochoerus } \\
\text { hidrochaeris }\end{array}$ & $x$ & $x$ & & $x$ & & & & \\
\hline Oso hormiguero blanco & Tamandua tetradactyla & $x$ & $\mathrm{x}$ & & & & & $x$ & \\
\hline Perezoso & Bradypus variegatus & $x$ & $x$ & & & & & & \\
\hline Perezoso-pelejo & Choloepus didactylus & $x$ & & & & & & & $\mathrm{x}$ \\
\hline Zorro & Cerdocyon thous & $x$ & $x$ & & & & & & \\
\hline Chosna & Potos flavus & $x$ & & & & $x$ & & & \\
\hline Tigre colorado & Puma concolor & $x$ & & & & & & & \\
\hline
\end{tabular}




\begin{tabular}{|l|l|l|l|l|l|l|l|l|l|}
\hline Tigre mariposo & Panthera onca & & $\mathrm{x}$ & $\mathrm{x}$ & & & & $\mathrm{x}$ & $\mathrm{x}$ \\
\hline Tigrillo & Leopardus sp. & & & $\mathrm{x}$ & & & & & \\
\hline Armadillo pequeño & Dasypus novemcinctus & $\mathrm{x}$ & $\mathrm{x}$ & & $\mathrm{x}$ & $\mathrm{x}$ & & & \\
\hline Armadillo grande & Priodontes maximus & $\mathrm{x}$ & & & & $\mathrm{x}$ & & & \\
\hline Ardilla & Sciureus sp. & $\mathrm{x}$ & & $\mathrm{x}$ & & & & & \\
\hline Chucha & Didelphis sp. & $\mathrm{x}$ & $\mathrm{x}$ & & & & & & \\
\hline Erizo & Coendu sp. & & $\mathrm{x}$ & & & & & & \\
\hline Nutria & Pteronura brasiliensis & $\mathrm{x}$ & & & & & & & \\
\hline
\end{tabular}

\begin{tabular}{|c|c|c|c|c|c|c|c|c|c|}
\hline \multicolumn{10}{|c|}{ AVES } \\
\hline Nombre Común & Especie & 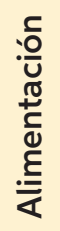 & 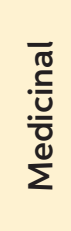 & $\frac{\mathscr{d}}{\frac{\tilde{d}}{\tilde{N}}}$ & 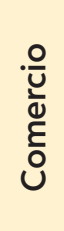 & 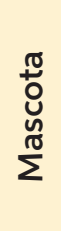 & 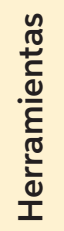 & 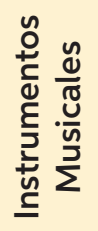 & 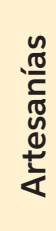 \\
\hline Pavón & Mitu tuberusum & $\mathrm{x}$ & & $x$ & & & & & $x$ \\
\hline Piuri & Crax globulosa & $x$ & & $x$ & $x$ & & & & $x$ \\
\hline Pava & Penelope jacquacu & $x$ & & & $\mathrm{x}$ & & & & \\
\hline Tente & Psophia crepitans & $x$ & & & & & & & \\
\hline Guacharaca & Ortalis guttata & $x$ & & & & & & & \\
\hline Perdiz & Tinamus major & $x$ & & & & & & & \\
\hline Panguana & Crypturellus undulatus & $x$ & & & & & & & \\
\hline Gallineta & Crypturellus variegatus & $x$ & & & & & & & \\
\hline Garza real & Ardea alba & & & $x$ & & & & & $x$ \\
\hline Pato aguja & Anhinga anhinga & $x$ & & & & & & & \\
\hline Piwicho frentiamarillo & $\begin{array}{l}\text { Brotogeris } \\
\text { santicthomae }\end{array}$ & & & & & $x$ & & & \\
\hline Piwicho & Brotogeris versicolurus & $x$ & & & & $x$ & & & \\
\hline Guacamaya & Ara macao & $x$ & & $x$ & & $x$ & & & $x$ \\
\hline Guamacaya azulamarilla & Ara ararauna & $x$ & & $x$ & & $x$ & & & $x$ \\
\hline Loro verde & Amazona festiva & $x$ & & $x$ & & $x$ & & & $x$ \\
\hline Lora cachetiamarilla & Amazona amazonica & & & & & $x$ & & & $\mathrm{x}$ \\
\hline Loro patilico & Pionites melanocephala & & & & & $x$ & & & \\
\hline Paloma & Patagioenas sp. & $x$ & & & & $x$ & & & \\
\hline Chulo & Coragyps atratus & & $x$ & & & & & & \\
\hline Gavilán churuquero & Rupornis magnirostris & & & $x$ & & & & & \\
\hline Águila real & Harpya harpija & & & $x$ & & & & & \\
\hline
\end{tabular}




\begin{tabular}{|c|c|c|c|c|c|c|c|c|c|}
\hline \multicolumn{10}{|c|}{ REPTILES } \\
\hline Nombre Común & Especie & 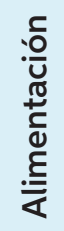 & 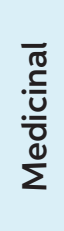 & $\frac{\tilde{d}}{\frac{\tilde{d}}{\tilde{N}}}$ & 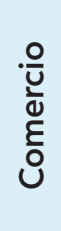 & 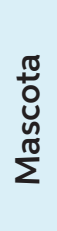 & 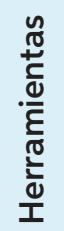 & 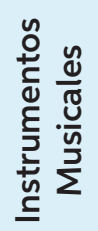 & 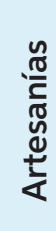 \\
\hline Caimán & Melanosuchus niger & $x$ & $x$ & $x$ & & & & & $x$ \\
\hline Babilla & Caiman crocodylus & $x$ & $x$ & & $x$ & $x$ & & & $x$ \\
\hline Morrocoy-motelo & Chelonoidis denticulata & $x$ & $x$ & $x$ & & $x$ & & $x$ & $x$ \\
\hline Charapa & Podocnemis expansa & $x$ & $x$ & $x$ & & $x$ & & & \\
\hline Taricaya & Podocnemis unifilis & $x$ & & $x$ & & $x$ & & & \\
\hline Cupiso & $\begin{array}{l}\text { Podocnemis } \\
\text { sextuberculata }\end{array}$ & $x$ & & & & & & & \\
\hline Matamata & Chelus fimbriatus & & $x$ & $x$ & & & & & \\
\hline Anaconda-Boa negra & Eunectes murinus & & $x$ & & & & & & \\
\hline Boa & Boa constrictor & & $x$ & & & & & & \\
\hline Coral & Micrurus sp. & & & & $\mathrm{x}$ & & & & \\
\hline Iguana & Iguana iguana & & $x$ & & & & & & \\
\hline
\end{tabular}

\begin{tabular}{|c|c|c|c|c|c|}
\hline \multicolumn{6}{|c|}{ ANFIBIOS } \\
\hline Sapo juanboy- gualo & Leptodactylus sp. & $x$ & & & $x$ \\
\hline \multicolumn{6}{|c|}{ INSECTOS } \\
\hline Mojojoi & $\begin{array}{l}\text { Rhynchophorus } \\
\text { palmarum }\end{array}$ & $x$ & $x$ & & \\
\hline Escarabajo & No identificada & & & & $x$ \\
\hline \multicolumn{6}{|c|}{ CARACOLES } \\
\hline Caracol & Pomacea diffusa & $x$ & & $x$ & \\
\hline
\end{tabular}

En general, la clase más utilizada es la de los mamíferos (Mammalia) con 35 especies, 48\% del total de especies, seguida por las aves con 23 especies, que corresponde al $32 \%$ de las especies utilizadas.

\section{PREFERENCIA DE ESPECIES PARA EL CONSUMO DE CARNE DE MONTE}

En general, la especie que más se consume es la que presenta mayor preferencia por la población encuestada, siendo la boruga o paca (Cuniculus paca), la especie con mayor preferencia de consumo en esta investigación, por su sabor, textura y variedad de platos que se pueden elaborar (Figura 3).

Sin embargo, cada comunidad presentó preferenciasdeconsumo deespecies diferentes, además de la boruga, que fue el común denominador para todas las localidades encuestadas. Por ejemplo, la comunidad de San Antonio de Los Lagos tuvo mayor preferencia por la carne de guara (Dasyprocta fuliginosa) y armadillo (Dasypus 


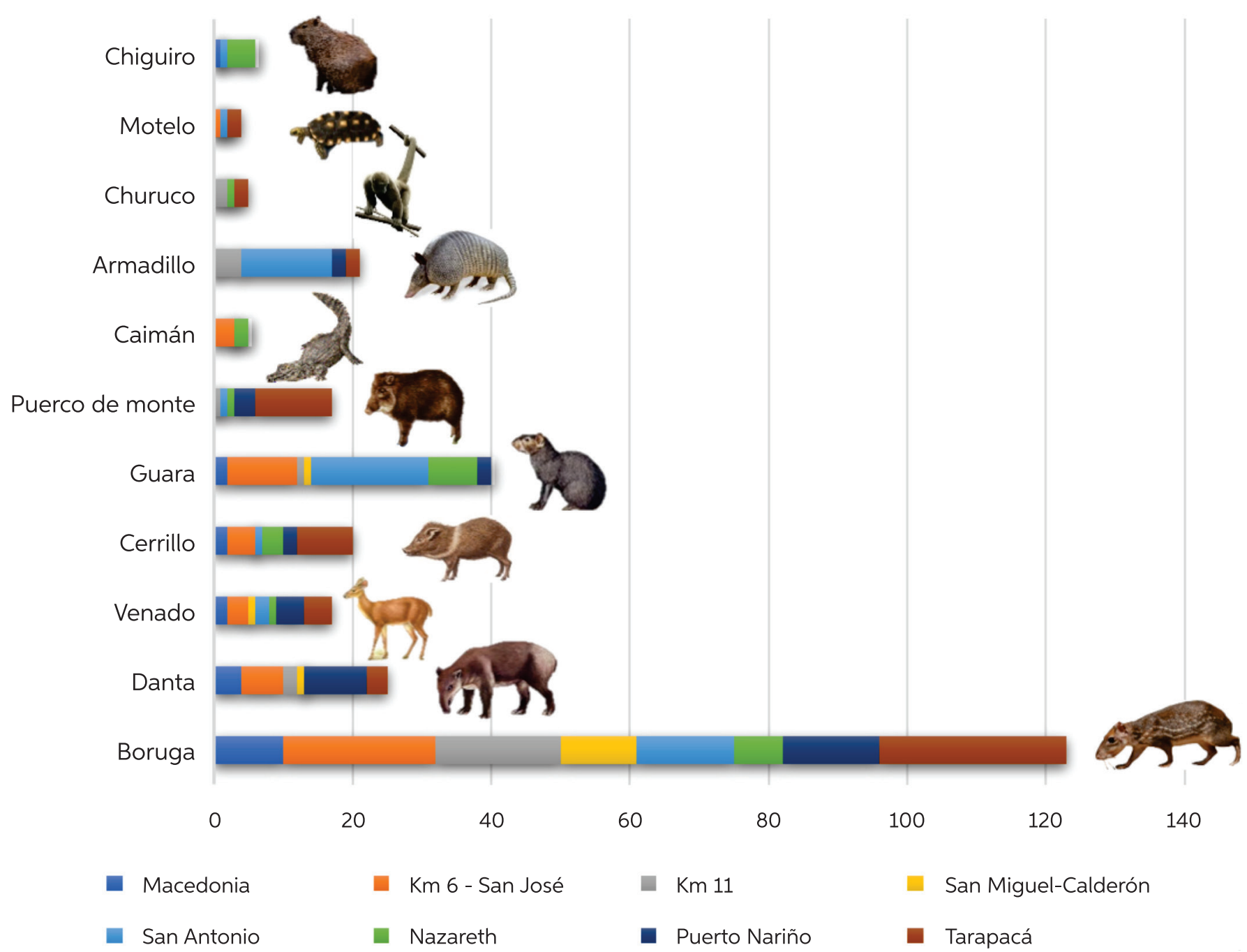

Figura 3. Especies por orden de preferencia para consumo de las comunidades encuestadas en el Trapecio Amazónico. llustraciones de animales obtenidos de internet.

novemcinctus), Tarapacá por el puerco de monte (Tayassu pecari) y el cerrillo (Pecari tajacu), Puerto Nariño por la danta (Tapirus terrestris), San José- Kilómetro 6 prefiere la carne de guara y danta, y Nazareth prefiere la guara y el chigüiro (Hydrochoerus hidrochaeris).

\section{FUENTES DE OBTENCIÓN Y PROCEDENCIA DE SUBPRODUCTOS DE FAUNA SILVESTRE}

Respecto a la procedencia de los subproductos que consumen (carne y huevos), la mayoría de los encuestados conoce el lugar de extracción, que es referenciado por el cazador. Los lugares de cacería son reconocidos como salados, pepeaderos y chagras que se encuentran dentro de la misma comunidad donde se encuentra el cazador y los consumidores.

La forma de obtención de los productos de fauna silvestre en el Amazonas, en la mayoría de casos es a través de la compra, seguido de la cacería. La actividad de trueque es frecuentada en el área no municipalizada de Tarapacá, en las demás comunidades no se practica. 
Frente al tema de la comercialización, son los mismos cazadores quienes se encargan de distribuirla en su comunidad. Para los asentamientos humanos ubicadas sobre el río Amazonas, es común el comercio de carne proveniente de sectores peruanos, que llegan a territorio colombiano a ofrecer sus productos. También se obtuvo información sobre lugares de comercialización de carne de animales silvestres como el Puerto de Leticia, Plaza de mercado de Tabatinga (Brasil), aunque reconocen la ilegalidad de esta práctica.

En Tarapacá, la carne y huevos son obtenidos en lugares cercanos y distribuidos en el sector a modo de pedido o puerta a puerta. Sin embargo, en esta zona, la forma más común de conseguir carne de animal silvestre es a modo de trueque, donde se intercambian productos o servicios solicitados por el cazador.

\section{OCURRENCIA DE CONSUMO DE CARNE DE MONTE}

En el Trapecio Amazónico, el consumo de carne de monte por parte de las comunidades indígenas y colonos no se hace de forma continua, son muy pocos los que afirman consumir este tipo de proteína de forma diaria. La mayoría consumen carne de monte por lo menos una o dos veces al mes o raramente. El mayor porcentaje de proteína animal se obtiene del pescado, además de consumir animales de granja como patos, gallinas $y$ algunas veces carne de ganado vacuno. Igualmente se registra la abstinencia de consumo de carne de animales silvestres bajo el ítem de "nunca" (siete encuestados manifestaron no consumir), debido a la palatabilidad o por concepción (antiguos consumidores) de conservación.

\section{TEMPORADA DE CONSUMO DE CARNE DE MONTE}

Se identificaron temporadas de consumo de especies silvestres, en las cuales se observa que la boruga (Cuniculus paca) es aprovechada de forma continua durante el año, a excepción del mes de abril, donde expresan los cazadores que se encuentra con cría. Igualmente pasa con la danta, la cual tiene dos temporadas de crías. Las demás especies muestran época de consumo

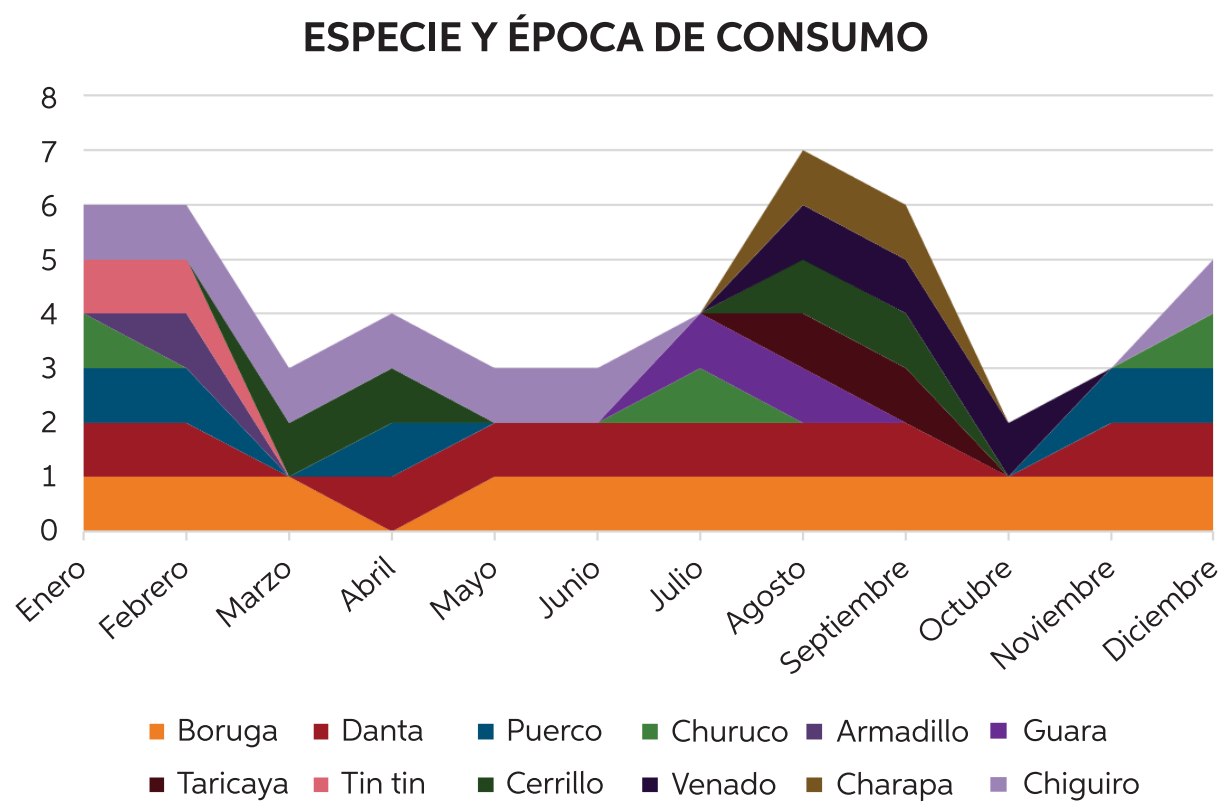

Figura 4. Temporadas de consumo de ciertas especies consumidas en el Trapecio Amazónico.

marcadas según la disponibilidad o cercanía a la comunidad (Figura 4).

La mayoría de los encuestados afirmó que, si bien hay épocas más fáciles para cazar, siempre hay animales disponibles, y que el cazador debe intensificar la jornada en búsqueda de ejemplares en temporadas bajas de caza. Igualmente existen diferencias en cuanto a los datos de temporadas 
de preñez-cría dados por los pobladores; sin embargo, la mayoría manifiesta que en lo posible, no cazan a hembras con crías.

\section{IDENTIFICACIÓN DE USOS MEDICINALES DE LA FAUNA SILVESTRE}

Para la identificación de usos medicinales, se registró la información brindada sobre qué animal utilizan, qué parte extraen o emplean, a qué enfermedad se le da tratamiento y cómo es su aplicación. En algunos casos, los encuestados presentaron información incompleta debido a que no tenían certeza o lo habían olvidado. En general, el grupo taxonómico con mayor número de especies aprovechadas con fines medicinales es la clase Mammalia, con 15 especies (60\%), seguida por Reptilia con siete especies (28\%).

\section{DISCUSIÓN}

El número de especies identificadas con algún uso por parte de las comunidades encuestadas coincide con estudios en comunidades indígenas de Mitú en zonas rurales con acceso a centros urbanos, donde se reportan 61 especies de
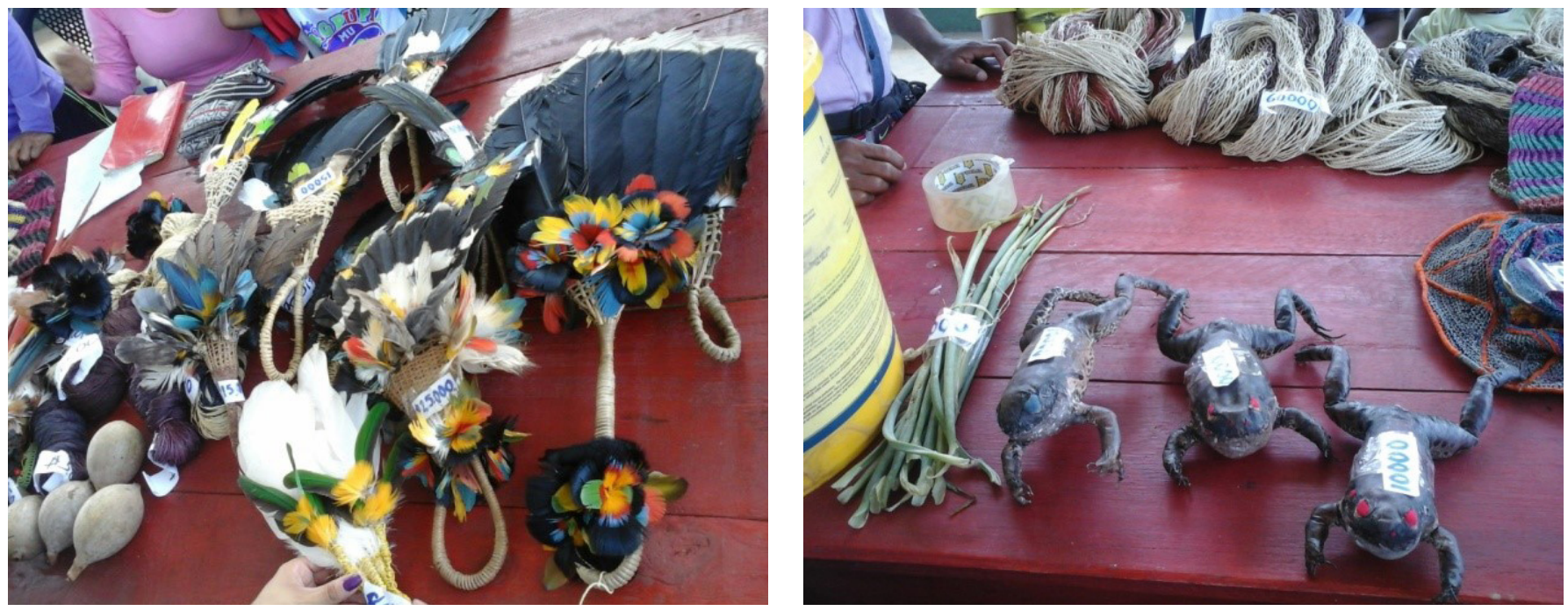

Figura 5. Adornos realizados con plumas de aves (izquierda) y sapo Gualo conservados (derecha) hechos por artesanos de Tarapacá. Fotos de la autora. 
la que se encuentran inmersas. El principal uso de animales es el alimenticio, y el aprovechamiento de los subproductos para fines medicinales y rituales mágico-religiosos. La caza de ciertas especies para uso como mascota es común en las comunidades, las cuales brindan compañía especialmente para las mujeres y niños.

El comercio de la fauna silvestre está enfocado principalmenteala venta dela carne paraconsumo y en menor medida a insumos para las artesanías (Figura 5); sin embargo, esta comercialización no ocurre abiertamente en zonas urbanas como Leticia, tal como se describe en Van Vliet et al (2014), y Sandrin et al., (2016). Esta situación de comercialización de carne de monte a nivel local, propicia la caza con fines comerciales (Pérez \& Ojasti, 1996), que tiene exigencias legales a diferencia de la caza de subsistencia. Algunos manifestaron comercializar animales vivos para ser usados como mascotas, a personas externas a sus comunidades. Los patrones de uso presentados en esta región amazónica colombiana son coincidentes con los patrones de uso de fauna silvestre reportados en otras comunidades de centro y Suramérica y que están relacionadas con la satisfacción de demanda de proteína animal de las comunidades (Pérez \& Ojasti, 1996, TCA, 1999, Nassar-Montoya 2000, Saldaña \& Rojas 2004, Racero et. al. 2008, Barrasa 2012, Puc Gil \& Retana 2012, Osorno et al. 2014).

En las comunidades donde se aplicaron las encuestas se pudo establecer que, entre mayor sea la cercanía con la comunidad colona y mejor sea el acceso a productos del mercado local, menos importancia tiene la fauna silvestre para las comunidades. Esta situación puede asociarse con el choque cultural que tienen las etnias indígenas con el hombre blanco, que ha generado cierta dependencia a los productos del mercado local para la alimentación de las familias indígenas, disminuyendo paulatinamente actividades como el trabajo en la chagra, la caza y la pesca, debido a que al tener poder de adquisición, es más asequible ir de compras y usar el servicio de medicina occidental, tal como lo muestra el informe de la gran expedición humana (Ruiz \& Valencia 2007).

Las comunidades se integran al mercado con más demanda que de oferta de productos, lo que genera un alto impacto en la alimentación debido a que se observa que las poblaciones recurren más al mercado para comprar productos según el mayor o menor acceso a centros urbanos, por lo cual es difícil encontrar en la actualidad alguna comunidad que consuma solo los alimentos que ellos producen, recolectan, cazan o pescan. Por esta razón, el mayor porcentaje de uso de animales silvestres se presentó en el área no municipalizada de Tarapacá, donde sus comunidades están asentadas principalmente sobre los ríos Putumayo y Cotuhé, y tienen menor disponibilidad de productos de granja y medicamentos comerciales, lo que permite continuar con sus hábitos tradicionales, a diferencia de las comunidades asentadas sobre la zona rural en el municipio de Leticia, como la comunidad San José (Kilómetro 6), comunidad del Kilómetro 11 y San Miguel.

El conocimiento de la fauna para el tratamiento de ciertas dolencias y enfermedades, es transmitido de padres a hijos o familiares cercanos. Entre mayor sea el uso de un animal para curar o mejorar la salud, más se difunde ese saber tradicional entre la comunidad, lo que se observó en las encuestas, donde se presentaron similitudes de usos de especies y formas de aplicación entre las comunidades estudiadas, tal es el caso de la manteca de caimán negro, que varias comunidades referenciaron utilizar para el tratamiento de afecciones respiratorias o el uso de la manteca de boa para los dolores musculares.

Las especies sobre las cuales existe mayor presión y posibilidades de disminución significativa de sus poblaciones, genera un riesgo 
de amenaza, lo que hace necesario, que se propongan estrategias que permitan el uso sostenible de las especies. Para poder plantear estrategias es necesario hacer estudios biológicos y etnobiológicos que permitan conocer la riqueza, diversidad y abundancia de especies en el Trapecio Amazónico, volúmenes de extracción y zonas de cacería para conocer el estado de conservación de la fauna silvestre en la región amazónica colombiana.

\section{PROPUESTA PARA LA GESTIÓN DE FAUNA SILVESTRE EN EL TRAPECIO AMAZÓNICO COLOMBIANO}

Actualmente el conocimiento sobre las poblaciones naturales en el Trapecio Amazónico, está limitado a estudios de línea base de diferentes universidades y caracterizaciones rápidas elaborados como requisito para trámites ambientales. Por lo cual, es necesario promover investigaciones que permitan conocer el estado real de poblaciones silvestres, su distribución y toda la información etno-zoológica como parte importante del conocimiento y manejo ancestral por parte de las comunidades indígenas del Trapecio Amazónico.

La gestión ambiental de la fauna silvestre en esta región amazónica está direccionada al fortalecimiento del conocimiento tradicional, que a su vez permite, el respectivo control social por parte de los indígenas, que sufren un continuo proceso de transformación cultural, donde dejan de lado sus raíces, las mismas que les permitió en un pasado cercano, hacer aprovechamientos sostenibles de los recursos sin depender del comercio externo. Aquí se presenta la apuesta a revitalizar, de revivir el conocimiento tradicional, de tal forma que se fortalezcan los mecanismos de orden social desde la cosmovisión indígena, la cual busca la convivencia armónica entre el hombre y la naturaleza.
Sin duda alguna, es importante revisar y ajustar esta propuesta acorde a los mecanismos de gestión establecidos y avalados por el Estado, no solo para realzar la importancia como cumplimiento de metas generales para el país en términos de la gestión de biodiversidad, sino además, para la consecución de recursos que permitan ejecutar estas estrategias y líneas de acción.

\section{A. ESTRATEGIA INTERCULTURAL DE MANEJO DE FAUNA SILVESTRE}

Esta estrategia consiste en reunir el conocimiento ancestral sobre la fauna silvestre y su aprovechamiento, información que tiende a desaparecer y que es necesaria para el ordenamiento ambiental del territorio y los procesos de gestión de la fauna silvestre en el trapecio Amazónico colombiano por parte del Estado. Se debe tener en cuenta dentro de esta estrategia los planes de vida, estatutos y planes de manejo ambiental existentes dentro del territorio, para garantizar que se acoja a sus necesidades y planteamientos establecidos.

\section{Líneas de acción}

A.1. Conocimiento ancestral

El rescate del conocimiento tradicional de los indígenas frente al aprovechamiento de la fauna silvestre es fundamental para asegurar la sostenibilidad del recurso; por esta razón es necesario definir los principios culturales de los pueblos indígenas en el manejo del territorio y sus recursos, de tal forma que se logre plasmar este conocimiento que sirva para su transmisión a las generaciones presentes y futuras.

Actividades:

- Diagnóstico. Responder las preguntas ¿Quiénes tienen y manejan el conocimiento 
ancestral de los animales y del bosque?, ¿Qué es lo que saben?, ¿Cómo hacen el aprovechamiento?, ¿con qué fin lo hacen?, ¿Cómo se hacía antes?

- Monitoreo participativo. Hacer un registro de uso de productos del bosque

- Investigación propia sobre recursos. Busca determinar cuáles son las especies útiles para los indígenas, qué temporadas son buenas, qué plantas se conservan en la chagra, cómo se ve el aprovechamiento y qué se desea para la comunidad.

\section{A.2. Fortalecimiento en la gobernanza del} territorio

Busca generar liderazgo frente a su propio territorio que permita la convivencia entre el hombre y la naturaleza, y en cumplimiento con los parámetros establecidos frente a los diferentes aprovechamientos de los recursos del bosque.

Actividades:

- Ordenación del territorio. La ordenación espacio-temporal del territorio de los pueblos indígenas se da a través de la definición de calendarios ecológicos para el manejo y uso de las especies silvestres (flora y fauna), respeto por las temporadas de veda, elaboración de mapas del territorio, definición de zonas de protección, zonas sagradas.

- Resolución de conflictos.

- Identificación de amenazas, debilidades, fortalezas y oportunidades

- Acuerdos voluntarios (internos o externos) de caza.

\section{B. ESTRATEGIA FORTALECIMIENTO DEL CONOCIMIENTO E INSTRUMENTOS DE APOYO}

\section{Líneas de acción}

B.1. Consolidación del conocimiento científico de fauna silvestre Como pilar de la gestión ambiental de fauna silvestre, se debe fomentar el conocimiento a través de investigaciones in situ, que además de permitir la toma de decisiones, permitan el aprovechamiento sostenible y actividades de zoocría.

Actividades:

- Investigaciones de estados poblaciones de especies representativas o con mayor valor de uso por parte de los pueblos indígenas.

- Caracterizaciones de flora y fauna

- Estudios base de cría de animales silvestres

\section{B.2. Educación ambiental}

El conocimiento adquirido debe ser socializado, así como las diferentes estrategias para minimizar las problemáticas ambientales que afectan la oferta natural y los servicios ecosistémicos del bosque. Así mismo, es necesario unificar conocimiento ancestral y conocimiento científico sobre fauna silvestre, que permitan tener una visión completa del estado real de los recursos, cómo utilizarlos y cómo recuperar la oferta natural.

Actividades:

- Talleres participativos de socialización de conocimiento tradicional y conocimiento científico acerca de fauna silvestre.

- Trabajo comunitario en la definición del calendario ecológico con resultados de los monitoreos comunitarios y las investigaciones científicas. 
- Encuentros interculturales entre los diferentes pueblos indígenas.

- Talleres con las escuelas en las comunidades indígenas.

\section{AGRADECIMIENTOS}

Esta investigación fue desarrollada bajo el contrato de prestación de servicios No. 0329 de 2015 con Corpoamazonia, en el marco del proyecto "Conservación de animales y ambientes silvestres de la Amazonía Colombiana (CASA) como un modelo de manejo in situ y ex situ, investigación y conservación de fauna silvestre, recurso hidrobiológico y sus hábitats objeto de presión antrópica en la jurisdicción de la Corporación para el Desarrollo Sostenible del Sur de la Amazonia- Corpoamazonia". Agradecimientos a las personas participantes de la investigación por su tiempo y disposición, así como a los curacas de las comunidades indígenas del Km 11, San Antonio, Nazareth, Macedonia y miembros del cabildo de la comunidad de San José-Kilómetro 6 que permitieron la entrada a sus territorios. A los compañeros de Corpoamazonia que colaboraron en la aplicación de las encuestas (Viviana Camelo, Paola Dávila, Xiomara Forero).

\section{BIBLIOGRAFIA CITADA}

Baptiste, L.; Polanco, R.; Quiceno, S.; Hernández, M. 2002. Fauna silvestre de Colombia: Historia económica y social de un proceso de marginalización. p. 295-340. En: Ulloa, A. (Ed). Rostros culturales de la fauna. Las relaciones entre los humanos y los animales en el contexto colombiano. Instituto Colombiano de Antropología e Historia - ICANH. Bogotá DC. Barrasa, S. 2012. Conocimiento y usos tradicionales de la fauna en dos comunidades campesinas de la Reserva de biosfera de La Encrucijada, Chiapas. Revista de Etnobiología 11(1): 16-28.

Cruz-Antia, D., Gómez, J. R. 2010. Aproximación al uso y tráfico de fauna silvestre en Puerto Carreño (Vichada, Colombia). Revista Ambiente y Desarrollo 14(26): 63-94.

De La Hoz, N. 2007. Uso de fauna silvestre por la comunidades indígenas. En: Ruiz S.; Sánchez, E.; Tabares, E.; Prieto, A.; Arias, J.C.; Gómez, R., ... Rodríguez, L. (Eds). Diversidad biológica y cultural del sur de la Amazonia ColombianaDiagnóstico. p. 356-358.

Millennium Ecosystem Assessment. 2005. Ecosystems and Human Well-being: Synthesis. Island Press. Washington, DC.

Nassar-Montoya, F. 2000. Actitud y pensamiento sobre la fauna silvestre en Colombia. En: Nassar-Montoya, F. \& Crane, R. (Eds). Actitudes hacia la fauna en Latinoamérica. p. 27-43.

Osorno, M.; Atuesta, N.; Jaramillo, L. F.; Sua, S.; Barona, A.; Roncancio, N. 2014. La despensa del Tiquié: Diagnóstico y manejo comunitario de la fauna de consumo en la Guayana colombiana.: Instituto Amazónico de Investigaciones Científicas SINCHI. Bogotá, D.C.

Pérez, E. M., \& Ojasti, J. (1996). La utilización de la fauna silvestre en la América Tropical y recomendaciones para su manejo sustentable en las sabanas. Ecotrópicos, 9(2), 71-82.

Piñuel, J. 2002. Epistemología, metodología y técnicas del análisis de contenido. Revista Estudios de Sociolingüística 3(1): 1-42.

Puc Gil, R.; Retana, O. 2012. Uso de la fauna silvestre en la comunidad Maya Villa de Guadalupe, Campeche, México. Revista Etnobiología 10(2): 1- 11.

Racero, J.; Vidal, C.; Ruiz, O.; Ballesteros, J. 2008. Percepción y patrones de uso de la fauna silvestre por las comunidades indígenas Embera-Katíos en la cuenca del río San Jorge, zona amortiguadora del PNN-Paramillo. 
Revista de Estudios Sociales (31): 118-131.

Ruiz, S.; Valencia, M. 2007. Contextualización del sur de la Amazonia Colombiana. In: Ruiz S.; Sánchez, E.; Tabares, A.; Prieto, J.; Arias, C.;Gómez, R. ... L. Rodríguez (Eds). Diversidad biológica y cultural del sur de la Amazonia Colombiana- Diagnóstico. p. 71-73.

Saldaña, J.; Rojas, T. 2004. Consumo de carne de monte y su importancia en la alimentación del poblador de Jenaro Herrera, Loreto Perú. Memorias: Manejo de fauna silvestre en Amazonia y Latinoamérica. 602-609 pp.

Sandrin, F., L'haridon, L., Vanegas, L., Ponta, N., Gómez, J., Revelo, J., . . . Van Vliet, N. (2016). Manejo comunitario de la cacería y de la fauna: Avances realizados por la asociación de cazadores airumakuchi en Puerto Nariño, Amazonas Colombia. Informe, CIFOR, Bogor, Indonesia.

SiB Colombia. 2016. Sistema de Información sobre Biovidersidad de Colombia. (www. sibcolombia.net/biodiversidad-en-cifras/). Acceso: 16/03/2017.

Tratado de Cooperación Amazónica. 1999. Conservación y uso de la fauna silvestre en áreas protegidas de la Amazonia. FAO, DGIS. Caracas. Van Vliet, N.; Quiceno-Mesa, M.P.; Cruz-Antia, D.; Yagüe, B. 2014. Carne de monte y seguridad alimentaria en la zona trifronteriza amazónica (Colombia, Perú y Brasil). CGIAR, USAID, CIFOR, Fundación SI, UFAM, Fundación Omacha. Bogotá, D.C.

Recibido: 23 de abril de 2018 Aceptado para publicación: 30 de mayo de 2018 